\title{
Multivariate associative patterns between the gut microbiota and large-scale brain network connectivity
}

Kohn, N. ${ }^{1,4 * \#, ~ S z o p i n s k a-T o k o v, ~ J .2,3 \#, ~ L l e r a, ~ A . ~}{ }^{1,4}$, Beckmann, C. ${ }^{1,4}$, Arias Vasquez, A. ${ }^{2,3 \#}$, Aarts, E. ${ }^{4, \#}$

1 Donders Institute for Brain, Cognition and Behaviour, Radboud University Medical Centre, Department of Cognitive Neuroscience, Geert Grooteplein Zuid 10, 6525GA Nijmegen, The Netherlands.

${ }^{2}$ Department of Psychiatry, Donders Institute for Brain, Cognition and Behaviour, Radboudumc, 6525 GA Nijmegen, The Netherlands.

3 Department of Human Genetics, Donders Institute for Brain, Cognition and Behaviour, Radboudumc, 6525 GA Nijmegen, The Netherlands.

${ }^{4}$ Centre for Cognitive Neuroimaging, Donders Institute for Brain, Cognition and Behaviour, Radboud University, Kapittelweg 29, 6525 EN Nijmegen, The Netherlands.

${ }^{\#}$ Equal contribution

Keywords: Linked ICA, gut microbiota, brain connectivity networks, fMRI

* Corresponding author:

Nils Kohn, tel.: 0031-243610866, email: n.kohn@donders.ru.nl, Kapittelweg 29, 6525 EN Nijmegen, The Netherlands 
Abstract:

Research on the gut-brain axis has accelerated substantially over the course of the last years. Many reviews have outlined the important implications of understanding the relation of the gut microbiota with human brain function and behavior. One substantial drawback in integrating gut microbiome and brain data is the lack of integrative multivariate approaches that enable capturing variance in both modalities simultaneously. To address this issue, we applied a linked independent component analysis (LICA) to microbiota and brain connectivity data.

We analyzed data from 58 healthy females (mean age $=21.5$ years). Magnetic Resonance Imaging data were acquired using resting state functional imaging data. The assessment of gut microbial composition from feces was based on sequencing of the V4 16S rRNA gene region. We used the LICA model to simultaneously factorize the subjects' large-scale brain networks and microbiome relative abundance data into 10 independent components of spatial and abundance variation.

LICA decomposition resulted in four components with non-marginal contribution of the microbiota data. The default mode network featured strongly in three components, whereas the two-lateralized fronto-parietal attention networks contributed to one component. The executive-control (with the default mode) network was associated to another component. We found the abundance of Prevotella genus was associated to the strength of expression of all networks, whereas Bifidobacterium was associated with the default mode and frontoparietal-attention networks.

We provide the first exploratory evidence for multivariate associative patterns between the gut microbiota and brain network connectivity in healthy humans, taking into account the complexity of both systems. 


\section{Introduction}

The gut-brain axis (GBA) is the bidirectional biochemical signaling that takes place between the gastrointestinal tract ( $\mathrm{Gl}$ tract) and the central nervous system (CNS)(1). The microbiota-GBA is used to describe the complex effects of the commensal gut bacteria (the microbiota) in the interplay between the gut and the brain. Recently, many studies have outlined the important implications of understanding the relation of the gut microbiota with human brain function and behavior. Several intermediary pathways have been proposed: specifically, bi-directional interactions between microbiota and the brain are plausible via modulation of vagal nerve activity, via neuromodulators or their precursors such as serotonin or tryptophan, via the Hypothalamic-Pituitary-Adrenal System (HPAaxis) and via interactions with the immune system (1-4).

In recent years, researchers aimed at elucidating these interactions, highlighting putative pathways, hormonal or immunological agents, and targeting the activity and interaction of certain bacterial strains (3). However, these studies have not taken into account the complexity and, especially, the full multivariate nature of both the brain and the gut microbiome.

One of these complex traits of the brain is the intrinsic connectivity between different brain regions. So far, studies assessing the relation between gut microbiome composition and intrinsic brain connectivity - with resting state fMRI - are rare, limited in rigor, and inconclusive (5). A recent study tested the effects of four weeks multi-strain probiotics supplementation (6). The authors report mild probiotics-induced changes in resting state connectivity of some of the ten networks tested. The strongest modulation was found in differences between the placebo $(n=15)$ and probiotics $(n=15)$ group, with the latter showing a relatively stronger increase in connectivity of the salience network to superior frontal brain regions. In another placebo-controlled trial of probiotics ( $n=20$ per group), Bifidobacterium longum influenced resting neural oscillations measured with magnetoencephalography (MEG), which correlated with enhanced vitality and reduced mental fatigue during a social stress induction task. Modulations of theta and alpha band oscillations by probiotics were localized in the frontal and cingulate cortex and supramarginal gyrus (7). However, these results (in relatively small samples) have not been related to probiotics-induced effects on gut microbiota composition.

A few studies did assess the relation between gut microbiome composition and intrinsic brain connectivity. One resting state fMRI study $(n=30)$, which included a subgroup of smokers, focused on the association of gut microbiota composition with insula connectivity and found its connection to several brain regions, such as occipital and lingual gyrus, frontal pole and cerebellar regions, to be associated with microbiota diversity and structure (8). Other exploratory region-of-interest (ROI) analyses did not reveal significant associations. Another resting state fMRI study ( $n=28$ vs 19) demonstrated that in end-stage renal disease, the integrity of the default mode network (DMN) was decreased along with alterations in the gut-microbiota composition (9). Taken together, these results are difficult to integrate and comprehend, as studies focus on one aspect of the modalities, such as connectivity from one particular ROI, or the gut-brain axis in targeted patient groups, or with different types of interventions. Most importantly, all previous studies have performed bivariate associations between one gut-microbiome composition measure and one brain connectivity measure (i.e. within one network or between two brain networks).

Indeed, in research, one approach to understand such complex systems is to try to elucidate the function of all its components sequentially and then to integrate interactions between a limited number of components. The opposite approach of investigation is to aim at integration at a macroscopic level. In this approach as many components as possible are sampled and patterns are 
investigated by dimensionality reduction. This has been attempted for the gut-brain axis very often narratively, in multiple reviews. Yet, no empirical attempt has been made so far to try to integrate the functions of the brain and the gut microbiome at a macroscopic level to determine associations between variance in macroscopic components of the two systems. In a similar approach, using data from the Human Connectome, researchers linked several lifestyle, demographic and psychometric measures in a positive-negative mode of brain connectivity (10).

Here, we aim to assess the relation between these two complex, multivariate modalities, focusing on canonically established brain networks in resting state that represent major modes of brain functioning in an unperturbed fashion (11). We asked if the inter-individual variability in abundance of gut microbiome genera was linked to variability in brain functional connectivity in canonical brain networks, when taking into account the full complexity of both.

One substantial methodological challenge is the multivariate and simultaneous integration of gut microbiome and brain data that enable capturing variance in both modalities simultaneously. To address this issue, we applied a linked independent component analysis $((12,13)$, LICA) to microbiota and brain connectivity data (Figure 1). LICA enables data reduction in several modalities simultaneously and thereby is able to demonstrate joint inter-individual variation patterns in different modalities. We chose to investigate four very well characterized and often replicated brain networks (11). We used this selection in previous work to investigate the impact of fasting on functional connectivity in rest (14). We limited our study to a set of four networks of interest (the lateralized fronto-parietal (left/right) attention networks, FPN; the executive control network, ECN; and the default mode network, DMN) due to their importance in the neuroimaging field, their comparatively clear and cognitive functional profile and their importance in mental disease or previous microbiome research $(10,11,14-16)$.

\section{A}
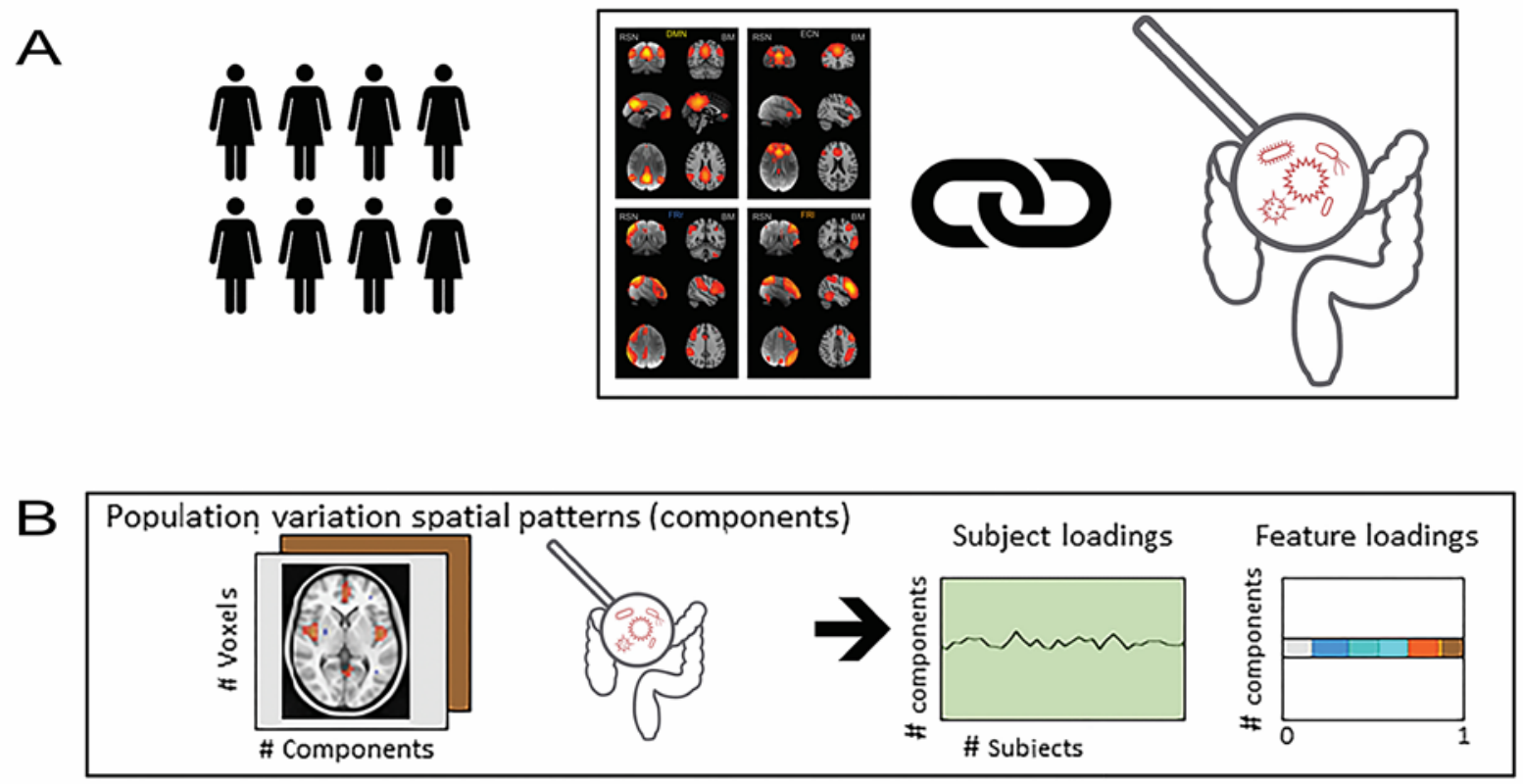

Figure 1. We linked functional brain connectivity in four well-established brain networks with relative abundance of human gut bacteria (microbiota). Panel A describes the Linked ICA that decomposed, simultaneously, the variability in functional connectivity of the four networks and the relative abundance of bacterial taxa (genera). This resulted in 10 components for which we have individual subject loadings as well as the loadings of each input feature depicted in panel B. The loadings represent voxel-wise association to the component in functional connectivity per network and genera-wise association to the component in the gut-microbiome. 


\section{Results}

For 58 subjects, the spatial template maps of right and left frontoparietal-attention networks (FPN), executive control (ECN), default mode network (DMN) (11) were projected onto the subjects resting state fMRI time-courses to create network maps per subject. Gut microbiome composition was based on sequencing of the V4 region of the 16S rRNA gene on the Illumina HiSeq platform. We used the LICA model to simultaneous factorize the subjects' brain networks and gut microbiome relative abundance into ten independent components $(12,13)$.

\section{Joint decomposition of brain networks and microbiome relative abundance}

From the 10 components, six showed a non-marginal (proportion $>0.2$ ) contribution on both the gut microbiota relative abundance and the brain connectivity patterns (Component 0, 1, 3, 4, 6 and 7ö Figure 2). From these six components, the first extracted component (component 0 ) was explained by a single subject, therefore, this component was disregarded for further analyses. Additionally, sanity checks on brain connectivity showed, for component 4, equal values for all voxels in the brain data. This renders interpretation of this component hardly possible and could potentially relate to residual noise being picked up and explained. This component was therefore also discarded.

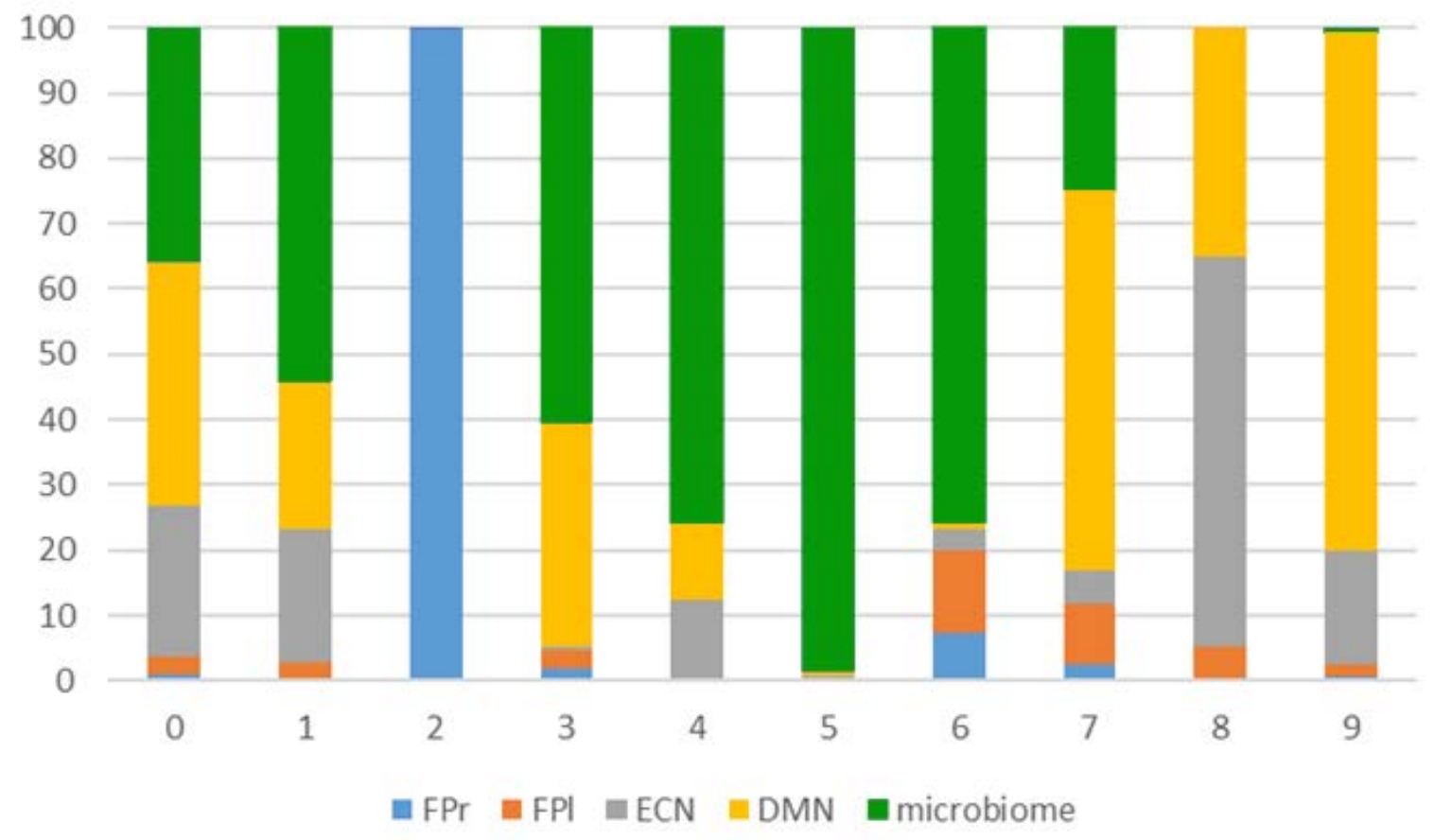

Figure 2 Decomposition of brain connectivity and microbiome. The plot shows the percentage of contribution per input modality. FPr and FPI are right and left fronto-parietal networks, DMN is default mode and ECN is executive control network.

We consequently investigated the association between brain connectivity and gut microbiota relative abundance in the four remaining components. We characterized each component by the contribution of the different modalities (proportion $>0.2$ for brain or microbiome). For each component, we plotted the brain-network and their voxel-wise loading and listed the bacterial genera that were nonmarginally associated with the component. For brain connectivity data, the z-maps from the Linked ICA were thresholded at $a \mathrm{z}>3$ for display purposes (see Neurovault: 
bioRxiv preprint doi: https://doi.org/10.1101/2020.08.25.266122; this version posted August 25, 2020. The copyright holder for this preprint

https://neurovault.org/collections/TRVFBPAB/ for z maps of the brain data four components). The zscores reflect how strongly a voxel covaries in connectivity with the respective input network. For microbiome data, high loadings reflect a robust covariation in relative abundance of a particular genus in that particular component. Similarly, we thresholded the microbiome loading at $z>2.3$ as they were more sparse compared to brain loadings (See Figure 3 for a visualization of these results and the DondersSharingCollection for the unthreshholded decomposition data).

The microbiota accounted for the majority of the variability that could be explained by component 1 , 3 and 6. Component 1 also has strong contributions from variability in the DMN and the ECN and the microbiota. Looking at the variability in each modality more deeply, the LICA enabled us to say which voxels show variation in functional connectivity to the respective network between subjects. For the microbiota, LICA gives an indication of between-subject variation in relative abundance of the gut microbiota genera for that component. For instance, ECN in component 1 varied between subjects in core hubs of the ECN, such as the dorsal paracingulate cortex, middle frontal and superior frontal gyri. The ECN largely consists of middle frontal and superior frontal gyri, paracingulate cortex and dorsal posterior parietal cortex $(11,17)$. This covariance in core hubs of the ECN can be interpreted as this component explaining functional connectivity strength, or the strength of the expression of the ECN in the subjects, and this strength of expression being related to variation in relative abundance of gut microbiota. The ECN has been demonstrated to overlap spatially with brain activity observed in cognitive control tasks, emotion tasks and response inhibition (11). Similarly, for the DMN, component 1 picked up on variability in the posterior core hub of the DMN (the posterior cingulate and retrosplenial cortex; 13,16$)$. The DMN is the large-scale brain network that was identified first and it is probably the most often studied of all so-called resting-state networks. DMN modulations have been implicated in a broad range of disorders (19-22). The most prominent feature of the DMN is its tasknegative nature; the areas of the DMN deactivate when an individual is engaged in most tasks (23-25). It has been associated to a broad range of cognitive processes such as self-referenced thought and self-monitoring (23), passive, broad attention $(20,26)$, auto-biographical memory retrieval $(27,28)$, imparting meaning in the current sensory input depending on prior experiences (29), mind-wandering and future thinking $(29,30)$ as well as homeostatic functions $(23,24,31,32)$. Prevotella_9 was more abundant and Blautia was less abundant with increasing between-subject functional connectivity of these hubs of the two networks. Component 2 had a contribution of over $50 \%$ from variability in microbiota abundance and the DMN. Bifidobacterium was more abundant and Prevotella_9 and Bacteroides were less abundant with increasing functional connectivity in anterior core hubs of the DMN. Component 6 had the strongest contribution of all components from microbiota of around $75 \%$, yet also explained variability in the two-lateralized fronto-parietal attention networks. As the name of these networks suggest, they encompass fronto-parietal brain regions, which are commonly and reliably associated to brain activity in attention tasks (11) and are modulated with varying degree of attention demand (33). The topology of the loading of these networks on this component overlaps with their common, canonical spatial profile in lateral frontal and parietal brain areas. Thus, again this component is associated to the between-subjects variation of the strength of expression of the lateralized attention networks. Prevotella_9, Bifidobacterium, genera belonging to Lachnospiracaceae family, and Faecalibacterium were more abundant and Christensenellacea_R-7_group was less abundant with stronger expression of the attention networks. Component 7 was associated to variability in the DMN again and to roughly $25 \%$ of the microbiota. The spatial pattern of betweensubject variation could be interpreted as elevated connectivity of the DMN to parts of the so-called salience network (such as dorsal anterior cingulate cortex (dorsal ACC) and ventrolateral prefrontal cortex (VLPFC)), which has in previous literature been associated with effects of elevated stress on DMN resting state connectivity $(15,16)$. Ruminocuccus_2 was more abundant and Blautia was less 
bioRxiv preprint doi: https://doi.org/10.1101/2020.08.25.266122; this version posted August 25, 2020. The copyright holder for this preprint (which was not certified by peer review) is the author/funder, who has granted bioRxiv a license to display the preprint in perpetuity. It is made available under aCC-BY-NC 4.0 International license.

abundant in individuals that showed this elevated connectivity pattern of DMN to dorsal ACC and VPLFC.

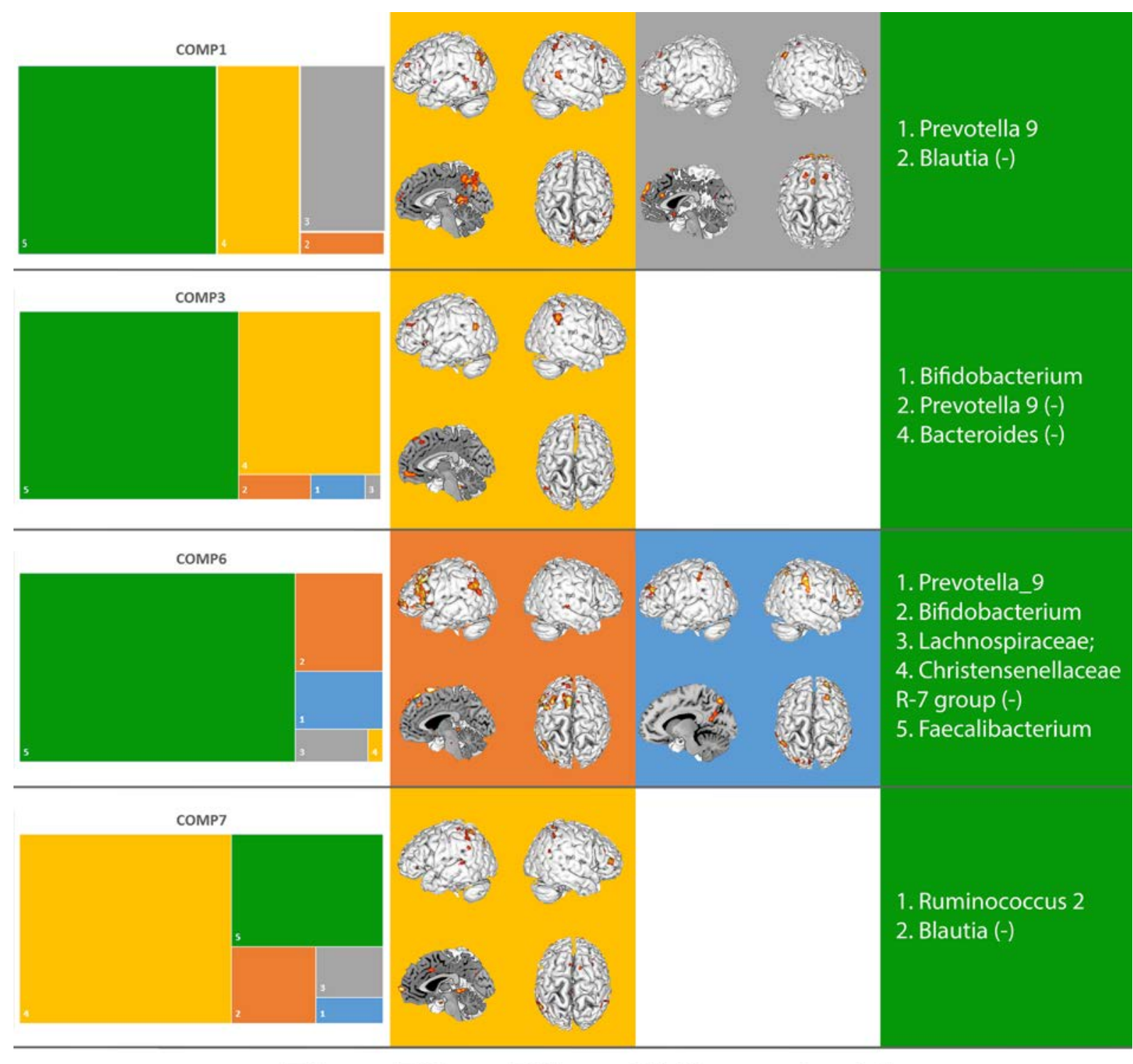

\section{- $\mathrm{FPr}=\mathrm{FPI}=\mathrm{ECN}=\mathrm{DMN}$ - microbiome}

Figure 3. Summary results of the contribution of each modality are shown in the first column (left). Second and third columns display the spatial project of the brain modalities, e.g. which voxels covary most strongly with covariation in other modalities (brain networks and microbiota abundance). Fourth column (right) displays the genera that show a covariance in abundance that is linked to covariance in the brain networks. The colors align with the modalities of the LICA (the four brain networks and the gut microbiota). For display purposes genera loading were cut at $z>2.3$ (for more details see the method section). See supplementary material for full list of genera loadings on the four components. 
bioRxiv preprint doi: https://doi.org/10.1101/2020.08.25.266122; this version posted August 25, 2020. The copyright holder for this preprint

\section{Discussion}

In this study, we provide the first evidence for multivariate associative patterns between the gut microbiota and brain network connectivity in healthy humans. We used a novel multivariate modality integration technique to explain inter-individual differences in brain connectivity in four canonical networks and the gut microbiota. We see our exploratory results as a map that could show high potential to guide future research on the relation of gut-brain interactions in a hypothesis-generating manner. We have linked ECN connectivity to an abundance of Prevotella_9 and Blautia; DMN connectivity to Prevotella_9, Blautia, Ruminococcus_2, Bifidobacterium, and Bacteroides; frontoparietal attention network connectivity to Prevotella_9, Bifidobacterium, Faecalibacterium, Christensenellacea_R-7_group, and certain genera belonging to Lachnospiracaceae. DMN connectivity that has been linked to stress is associated with Ruminocuccus_2 and Blautia. The spatial associations in the components to core hubs of the respective networks can be seen as a conceptual validation of our approach $(11,16,34)$. Furthermore, we observe the between-subject variation in functional connectivity in core hubs of the respective networks in three of the four components as a link between an individual's connectivity strength and the relative abundance of certain microbiota. These findings can be taken as an indication that certain microbial genera are associated with the normal expression of all four canonical resting state networks and their natural variation between healthy subjects.

On the side of the bacterial genera that were associated with brain network connectivity, we found that inter-individual variation in abundance of the Bifidobacterium genus was prominently contributing to two of our four identified components. Variation in abundance of Bifidobacteria were associated with increased connectivity of the medial prefrontal cortex of the DMN and parietal regions and in another component with modulated connectivity of the core hubs of the fronto-parietal attention network. The Bifidobacterium genus is probably one of the most noticeable targets in current gut-brain axis research $(2,35,36)$. This strong focus is potentially related to a landmark study, which showed that germ-free mice have altered HPA-axis function, and this altered HPA activity was reversed by colonization with a Bifidobaterium (1). Bifidobacteria are one of the most important and abundant genera during development and have been associated with decreased levels of inflammation in human development (37). Bifidobacterium longum, a strain commonly used in probiotic products, influenced resting neural activity that correlated with enhanced vitality and reduced mental fatigue during a social stress induction task (7). The medial prefrontal cortex and the DMN have been related to autobiographic and episodic memory or prior knowledge structures(38), which fits to findings of the link between increased Bifidobacteria after interventions and elevated verbal episodic memory(6). Furthermore, Bagga and colleagues also found altered functional connectivity of the DMN after probiotic use (including $B$. longum) (6). Component 3 might therefor partially reflect episodic memoryrelated modulations in Bifidobacteria and DMN connectivity. A probiotics trial with Bidfidobacterium longum using electrophysiological resting state brain recordings found evidence for an association of increased frontal midline mobility and improved memory after probiotics consumption compared to placebo (39). The authors related the brain recordings to attention-related brain activity. Moreover, the fact that out of $>50$ genera that featured in our analysis, Bifidobacteria featured in two of the four components both underscores their putative influence in the gut-brain interaction and the validity of our integrative approach. In summary, we found evidence for a relation of Bifidobacteria abundance to attention- and potentially memory-related brain network activity at rest.

For component 7, the spatial patterns of association were similar to results showing an alerted state of the DMN after social stress induction (15). In data using a similar paradigm, Bifidobacterium longum modulated activity in similar regions that were influenced by social stress and also in the hippocampus, a region that is part of the DMN (7). This pattern particularly varied with abundance in Blautia and 
bioRxiv preprint doi: https://doi.org/10.1101/2020.08.25.266122; this version posted August 25,2020 . The copyright holder for this preprint

Ruminococcus 2. Although Bifidobacterium did not covary with this component, the Bifidobacterium intake might have indirectly affected DMN connectivity in stress, potentially via modulation of the abundance of Ruminococcus 2 and Blautia. Indeed, the pre-existing levels of Blautia and Ruminococcae correlated with the metabolic outcomes of a Bifidobacterium-targeting prebiotic intervention in obese patients(40). Furthermore, Blautia has been found to be the only genus to be enriched in depressionmodel rats(41) and both Blautia and Ruminococcae correlated with stress-related depression-like behaviour in mice(42). In summary, variations in Blautia and Ruminococcus 2 abundance might relate to stress-induced modulation of DMN connectivity.

The association of Components 1, 3 and 6 with Prevotella_9 is interesting as this genus has been previously involved in psychiatric disorders, cognition and brain connectivity changes. For example, in autism spectrum disorder (ASD), which is characterized by atypical brain network organization (including DMN and ECN, as in component 1) (43), a higher relative abundance of Prevotella (and Bifidobacterium) has been linked with a beneficial effect of Microbiota Transfer Therapy (44). Accordingly, lower relative abundance of Prevotella has been associated with psychiatric disorders like ADHD in children (45), Parkinson's disease (46), and ASD (47). Furthermore, the gut-brain axis may play a role in the disturbed executive functioning in ASD (for a review, see (48)). Our finding of a positive correlation of Prevotella with DMN and ECN functioning and also fronto-parietal attention network modulations support these results. Previous work showed a link between gut microbiota and restingstate functional connectivity, as assessed here $(9,49)$. Interestingly, in one study assessing bivariate relationships, Prevotella and Bacteroides were associated with insular connectivity (8). The insula has not only been discussed as part of the salience network, but also as an important component of the general task positive network $(50,51)$. In our case, both of the Prevotella and Bacteroides genera were negatively associated with $\mathrm{DMN}$ in component 3 . As the $\mathrm{DMN}$ is thought to be anti-correlated with the task positive network, our finding is in line with previous results (50). Prevotella seem to be associated to healthy modulation in brain connectivity related to attention, cognitive control, episodic memory and a range of other psychological functions.

Our design and approach have limitations in the interpretation of the results. First, these findings are necessarily limited to more common genera. We capped our analysis at genera that are at least detectable in $30 \%$ of our subjects. Genera with lower occurrence rates in individuals might have unequally strong leverage on the LICA. As a consequence of this methodological choice, we cannot exclude an overestimation of the loadings for the more common taxa (given the sample size) and we cannot assess the rare genera and their association with brain network connectivity.

Second, the selection of brain networks was motivated by their role in cognition specifically to highlevel cognitive constructs such as attention and cognitive control and their relevance in the literature . While we perceive this selection as well-motivated and we have demonstrated their sensitivity (14), it is a subjective pre-selection. We might not cover other cognitive processes and associated brain networks equally well. Nevertheless, due to the limited power in our sample and for the advantage of choosing networks that are more readily interpreted, we chose to limit our selection of brain networks to these four.

Third, we investigated a very homogenous, healthy and young group of only female participants. Although this naturally limits the generalizability of the results, we believe that our data still serves an orientating purpose and is therefore valuable. In replication attempts, this homogeneity and special characteristic of our sample should be considered. We would like to reiterate that we see a strong need to replicate the current results in larger and more diverse samples. 
In summary, we provided the first evidence for multivariate associative patterns between large-scale brain network functional connectivity of four very well-established brain networks and the relative abundance of gut microbiota in a sample of healthy female individuals. This link provides a map for future research, involving the full complexity of both measures into account. For example, interventions targeting improvement in attention (for example in neurodevelopmental disorders) could investigate the influence on the bacterial genera associated to the attention networks. Moreover, it can provide a roadmap to investigate how the effect of probiotic intervention trials with compounds that benefit certain genera could explicitly test modulations in cognitive functions associated to the brain networks we investigated or to brain connectivity itself. Furthermore, future research might investigate the mechanistic nature of our multivariate associative patterns and aim to assess the generalizability to other healthy samples as well as their potential disruption in the diseased brain. 
bioRxiv preprint doi: https://doi.org/10.1101/2020.08.25.266122; this version posted August 25, 2020. The copyright holder for this preprint

Material and Methods

Sample

We analyzed pre-intervention data from a probiotics intervention study on 64 healthy female participants (mean age $=21.5(0.45)$ years) (52). In total, 58 of the 64 participants were included in the analyses. Six participants were excluded from the final analyses, due to high depression scores $(\mathrm{N}=1)$, missing feces samples $(N=2)$, and movement exceeding $4 \mathrm{~mm}$ between acquisitions $(n=3)$. For more detailed characteristic of the samples and exclusion criteria as well as the ethical declaration, please see the Material and Methods section of Papalini et al. (52). Briefly, participants with relevant medical history of e.g. psychiatric and/or gastrointestinal disorder were excluded. Also, use of antibiotics and diet like e.g. vegan diet were part of the exclusion criteria.

fMRI data acquisition

Participants were screened for compatibility to magnetic resonance imaging (MRI). MRI data were acquired using a 3T MAGNETOM Prisma system, equipped with a 32-channel head coil. After three short task-related fMRI scans (see Papalini et al.), nine minutes of resting state fMRI was acquired. 3D echo planar imaging (EPI) scans using a $\mathrm{T} 2 *$ weighted gradient echo multi-echo sequence (Poser, Versluis et al. 2006) were acquired (voxel size $3.5 \times 3.5 \times 3 \mathrm{~mm}$ isotropic, TR $=2070 \mathrm{~ms}$, TE = $9 \mathrm{~ms} ; 19.25$ $\mathrm{ms} ; 29.5 \mathrm{~ms} ; 39.75 \mathrm{~ms}$, FoV $=224 \mathrm{~mm}$ ). The slab positioning and rotation (average angle of 14 degrees to $A C$ axis) optimally covered both prefrontal and deep brain regions. Subjects were instructed to lie still with their eyes open and refrain from directed thought. A whole-brain high-resolution T1-weighted anatomical scan was acquired using a MPRAGE sequence (voxel size $1.0 \times 1.0 \times 1.0$ isotropic, TR $=2300$ $\mathrm{ms}, \mathrm{TE}=3.03 \mathrm{~ms}, 192$ slices).

MRI data preprocessing: FSL (FMRIB, University of Oxford, UK; www.fmrib.ox.ac.uk/fsl; (Jenkinson et al., 2012) was used for pre-processing, data-denoising, and generation of subject-specific network maps. Pre-processing steps included three-dimensional movement correction, and spatial smoothing using a $5 \mathrm{~mm}$ full-width at half maximum (FWHM) Gaussian kernel to reduce inter-subject variability and a high-pass filter $(>0.007 \mathrm{~Hz})$ was applied. All pre-processing steps, except temporal filtering, were conducted before AROMA data denoising $(53,54)$. Briefly, ICA-AROMA is designed to identify motionrelated artifacts by matching single subject ICA components to four robust and standardized features. The data is denoised by linear regression of ICA components identified as noise by AROMA and subsequently the high pass filter was applied. Prior to all group analyses, data were normalized to MNI space and re-sampled to $2 \mathrm{~mm}^{3}$ resolution using FMRIB's Nonlinear Image Registration Tool (FNIRT).

Generation of subject-specific functional connectivity maps

Dual (spatial and temporal) regression was used to generate subject-specific spatial maps of wellstudied, canonical large-scale brain networks(11) from the individuals' data. The z-maps of these networks were temporally concatenated in one 4D file and used as input for the dual regression. These maps were used in a linear model fit against the individual fMRI data, resulting in the subject-specific temporal dynamics. Subsequently, these time-course matrices are employed in a linear model fit against the subject's fMRI data set to estimate subject-specific spatial maps. From these subject-wise expressions of the 10 networks, we selected four networks of interest (the left and right lateralized fronto-parietal attention networks, FPN; the executive control network, ECN; and the default mode network, DMN), due to their importance in the neuroimaging field, their comparatively clear functional profile and their importance in mental disease or previous microbiome research $(10,11,14-16)$. The different spatial maps for all participants are combined into a single 4D file per target network. In this way, we generated four files for the four respective networks of interest that contain one spatial z- 
bioRxiv preprint doi: https://doi.org/10.1101/2020.08.25.266122; this version posted August 25, 2020. The copyright holder for this preprin

map per subject that indicates for each voxel the connectivity strength of the respective network in that individual. These four network files were used as inputs to the Linked-ICA.

\section{Gut microbiome analysis}

Fecal samples were collected by using OMNIgene•GUT kit (DNAGenotek, Ottawa, CA) (55). Collected fecal samples were transported to the laboratory and aliquoted into $1.5 \mathrm{~mL}$ Eppendorf tubes and stored at $-80^{\circ} \mathrm{C}$ for microbiome analysis. DNA was isolated from the fecal pellets using the Maxwell ${ }^{\circledast}$ 16 Instrument (Promega, Leiden, The Netherlands) as described previously (56). Briefly, in the 2-step PCR protocol the $16 \mathrm{~S}$ rRNA gene V4 variable region was targeted by using 515F (GTGYCAGCMGCCGCGGTAA) and 806R (GGACTACNVGGGTWTCTAAT) primers, and unique barcodes were used to characterize a mixture of bacteria. Sequencing was performed on the Illumina HiSeq PE300 platform by GATC Biotech AG (Konstanz, Germany). The sequences were processed using NGTax (57) analysis pipeline as described previously (58). This resulted in an Operational Taxonomical Unit (OTU) table containing 844 OTUs. We applied a prevalence-filtering at the genus level, selecting genera present in at least $30 \%$ of the samples. After this step, the OTU-table containing 644 OTUs was used for the downstream analyses. The gut microbiome composition tables at the phylum and genus taxonomic levels were provided by the 'phyloseq' package available in R (59).

Linked analyses

We used the Linked-ICA model (13) to simultaneous factorize the functional network maps (of ECN, FPNs and DMN) and the microbiome data of 58 subjects into independent sources (or components) of variation. In the brain networks, spatial variation was explained; while in the microbiome data, variation in relative abundance of bacterial genera was explained. In brief, Linked-ICA is an extension of Bayesian ICA (60) to multiple input sets, where all individual ICA factorizations are linked through a shared common mixing matrix that reflect the subject-wise contribution to each component (Figure 1).

This operation is represented in Figure 1. Factorization provides a set of spatial maps (one per feature modality and component), a vector of feature loadings that reflects the degree to which the component 'represents' the different modalities, and a vector that reflects the contribution of the individual subject to a given component. All mathematical derivations involved in the Linked-ICA factorization can be found in the original paper describing the original algorithm (13). Further details and code implementing each feature extraction procedure as well as the Linked-ICA factorization are publicly available at (61). Given the sample size, we forced a 10 components solution. We disregarded components estimated with marginal (proportion $<0.2$ ) contribution of the microbiome or brain networks, respectively.

Data will be shared via the DondersSharingCollection (link to follow).

\section{Acknowledgements}

The study was supported by the Dutch Ministry of Economic Affairs under the TKI Life Science and Health, project LSHM15034. CB and AL have received funding from the Innovative Medicines Initiative 2 Joint Undertaking under grant agreement No 777394 for the project AIMS-2-TRIALS. This Joint Undertaking receives support from the European Union's Horizon 2020 research and innovation programme and EFPIA and AUTISM SPEAKS, Autistica, SFARI. This work was further supported by the European Union Horizon 2020 programme CANDY (Grant Agreement No. 847818). EA received funding from the European Research Council (ERC_StG2019_852189). JST and AAV have received 
bioRxiv preprint doi: https://doi.org/10.1101/2020.08.25.266122; this version posted August 25, 2020. The copyright holder for this preprint (which was not certified by peer review) is the author/funder, who has granted bioRxiv a license to display the preprint in perpetuity. It is made available under aCC-BY-NC 4.0 International license.

support from the European Union's Horizon 2020 research and innovation programme under grant agreement No 728018 (Eat2beNICE). 


\section{Reference}

1. N. Sudo, et al., Postnatal microbial colonization programs the hypothalamic-pituitary-adrenal system for stress response in mice. J. Physiol. (Lond.) 558, 263-275 (2004).

2. J. F. Cryan, T. G. Dinan, Mind-altering microorganisms: the impact of the gut microbiota on brain and behaviour. Nat Rev Neurosci 13, 701-712 (2012).

3. J. F. Cryan, et al., The Microbiota-Gut-Brain Axis. Physiol. Rev. 99, 1877-2013 (2019).

4. C. Fülling, T. G. Dinan, J. F. Cryan, Gut Microbe to Brain Signaling: What Happens in Vagus.... Neuron 101, 998-1002 (2019).

5. P. Liu, G. Peng, N. Zhang, B. Wang, B. Luo, Crosstalk Between the Gut Microbiota and the Brain: An Update on Neuroimaging Findings. Front Neurol 10, 883 (2019).

6. D. Bagga, et al., Influence of 4-week multi-strain probiotic administration on resting-state functional connectivity in healthy volunteers. Eur J Nutr 58, 1821-1827 (2019).

7. H. Wang, C. Braun, E. F. Murphy, P. Enck, Bifidobacterium longum $1714^{\mathrm{TM}}$ Strain Modulates Brain Activity of Healthy Volunteers During Social Stress. Am. J. Gastroenterol. 114, 1152 1162 (2019).

8. K. Curtis, et al., Insular resting state functional connectivity is associated with gut microbiota diversity. Eur. J. Neurosci. 50, 2446-2452 (2019).

9. Y. F. Wang, et al., The gut microbiota-inflammation-brain axis in end-stage renal disease: perspectives from default mode network. Theranostics 9, 8171-8181 (2019).

10. S. Smith, et al., A positive-negative mode of population covariation links brain connectivity, demographics and behavior. Nat Neurosci 18, 1565-1567 (2015).

11. S. M. Smith, et al., Correspondence of the brain's functional architecture during activation and rest. PNAS 106, 13040-13045 (2009).

12. A. Llera, T. Wolfers, P. Mulders, C. F. Beckmann, Inter-individual differences in human brain structure and morphology link to variation in demographics and behavior. eLife 8, e44443 (2019).

13. A. R. Groves, C. F. Beckmann, S. M. Smith, M. W. Woolrich, Linked independent component analysis for multimodal data fusion. Neurolmage 54, 2198-2217 (2011).

14. S. Orfanos, et al., Investigating the impact of overnight fasting on intrinsic functional connectivity: a double-blind fMRI study. Brain Imaging and Behavior 12 (2017).

15. B. Clemens, et al., Alerted default mode: functional connectivity changes in the aftermath of social stress. Sci Rep 7, 1-9 (2017).

16. E. J. Hermans, M. J. A. G. Henckens, M. Joëls, G. Fernández, Dynamic adaptation of large-scale brain networks in response to acute stressors. Trends in neurosciences 37, 304-14 (2014).

17. N. Kohn, E. J. Hermans, G. Fernández, Cognitive benefit and cost of acute stress is differentially modulated by individual brain state. Soc Cogn Affect Neurosci 12, 1179-1187 (2017). 
18. J. R. Andrews-Hanna, J. S. Reidler, J. Sepulcre, R. Poulin, R. L. Buckner, Functional-anatomic fractionation of the brain's default network. Neuron 65, 550-62 (2010).

19. M. E. Raichle, The Brain's Default Mode Network. Annual Review of Neuroscience 38, 433-447 (2015).

20. R. L. Buckner, J. R. Andrews-Hanna, D. L. Schacter, The brain's default network: anatomy, function, and relevance to disease. Annals of the New York Academy of Sciences 1124, 1-38 (2008).

21. J. R. Andrews-Hanna, J. Smallwood, R. N. Spreng, The default network and self-generated thought: component processes, dynamic control, and clinical relevance. Annals of the New York Academy of Sciences 1316, 29-52 (2014).

22. S. J. Broyd, et al., Default-mode brain dysfunction in mental disorders: A systematic review. Neuroscience \& Biobehavioral Reviews 33, 279-296 (2009).

23. D. A. Gusnard, E. Akbudak, G. L. Shulman, M. E. Raichle, Medial prefrontal cortex and selfreferential mental activity: relation to a default mode of brain function. Proc Natl Acad Sci U S A 98, 4259-4264 (2001).

24. M. E. Raichle, et al., A default mode of brain function. Proc Natl Acad Sci U S A 98, 676-682 (2001).

25. G. Shulman, J. Fiez, M. Corbetta, Common blood flow changes across visual tasks: II. Decreases in cerebral cortex. Journal of Cognitive ..., 648-663 (1997).

26. M. E. Raichle, A. Z. Snyder, A default mode of brain function: A brief history of an evolving idea. Neurolmage 37, 1083-1090 (2007).

27. K. Mevel, et al., Age effect on the default mode network, inner thoughts, and cognitive abilities. Neurobiology of Aging 34, 1292-1301 (2013).

28. C. Sestieri, M. Corbetta, G. L. Romani, G. L. Shulman, Episodic memory retrieval, parietal cortex, and the default mode network: functional and topographic analyses. The Journal of neuroscience : the official journal of the Society for Neuroscience 31, 4407-20 (2011).

29. M. Bar, The proactive brain: using analogies and associations to generate predictions. Trends in Cognitive Sciences 11, 280-289 (2007).

30. M. F. Mason, et al., Wandering Minds: The Default Network and Stimulus-Independent Thought. Science 315 (2007).

31. M. E. Raichle, The restless brain: how intrinsic activity organizes brain function. Philosophical Transactions of the Royal Society B: Biological Sciences 370, 20140172-20140172 (2015).

32. N. Kohn, et al., In a sweet mood? Effects of experimental modulation of blood glucose levels on mood-induction during fMRI. Neurolmage 113, 246-56 (2015).

33. S. E. Petersen, M. I. Posner, The Attention System of the Human Brain: 20 Years After. Annual Review of Neuroscience 35, 73-89 (2012). 
34. V. Menon, Large-scale brain networks and psychopathology: a unifying triple network model. Trends in Cognitive Sciences 15, 483-506 (2011).

35. J. A. Foster, L. Rinaman, J. F. Cryan, Stress \& the gut-brain axis: Regulation by the microbiome. Neurobiology of Stress 7, 124-136 (2017).

36. K. V. Sandhu, et al., Feeding the microbiota-gut-brain axis: diet, microbiome, and neuropsychiatry. Translational Research 179, 223-244 (2017).

37. C. de Weerth, Do bacteria shape our development? Crosstalk between intestinal microbiota and HPA axis. Neurosci Biobehav Rev 83, 458-471 (2017).

38. N. C. J. Müller, et al., Medial prefrontal decoupling from the default mode network benefits memory. Neurolmage 210, 116543 (2020).

39. A. P. Allen, et al., Bifidobacterium longum 1714 as a translational psychobiotic: modulation of stress, electrophysiology and neurocognition in healthy volunteers. Translational Psychiatry 6 , e939-e939 (2016).

40. J. Rodriguez, et al., Discovery of the gut microbial signature driving the efficacy of prebiotic intervention in obese patients. Gut (2020) https:/doi.org/10.1136/gutjnl-2019-319726 (August 10, 2020).

41. L. Jianguo, J. Xueyang, W. Cui, W. Changxin, Q. Xuemei, Altered gut metabolome contributes to depression-like behaviors in rats exposed to chronic unpredictable mild stress.

Translational Psychiatry 9, 1-14 (2019).

42. T. Tian, et al., Clostridium butyricum miyairi 588 has preventive effects on chronic social defeat stress-induced depressive-like behaviour and modulates microglial activation in mice. Biochemical and Biophysical Research Communications 516, 430-436 (2019).

43. A. E. Abbott, et al., Patterns of Atypical Functional Connectivity and Behavioral Links in Autism Differ Between Default, Salience, and Executive Networks. Cereb Cortex 26, 4034-4045 (2016).

44. D.-W. Kang, et al., Long-term benefit of Microbiota Transfer Therapy on autism symptoms and gut microbiota. Scientific Reports 9, 1-9 (2019).

45. A. Prehn-Kristensen, et al., Reduced microbiome alpha diversity in young patients with ADHD. PLoS One 13 (2018).

46. S. Gerhardt, M. H. Mohajeri, Changes of Colonic Bacterial Composition in Parkinson's Disease and Other Neurodegenerative Diseases. Nutrients 10 (2018).

47. L. K. H. Ho, et al., Gut microbiota changes in children with autism spectrum disorder: a systematic review. Gut Pathog 12, 6 (2020).

48. P. Roman, L. Rueda-Ruzafa, D. Cardona, A. Cortes-Rodríguez, Gut-brain axis in the executive function of austism spectrum disorder. Behavioural Pharmacology 29, 654-663 (2018).

49. A. C. Tengeler, et al., Gut microbiota from persons with attention-deficit/hyperactivity disorder affects the brain in mice. Microbiome 8, 44 (2020). 
50. X. Di, B. B. Biswal, Modulatory interactions between the default mode network and task positive networks in resting-state. PeerJ 2 (2014).

51. M. D. Fox, et al., The human brain is intrinsically organized into dynamic, anticorrelated functional networks. Proc Natl Acad Sci U S A 102, 9673-9678 (2005).

52. S. Papalini, et al., Stress matters: Randomized controlled trial on the effect of probiotics on neurocognition. Neurobiology of Stress 10, 100141 (2019).

53. R. H. R. Pruim, et al., ICA-AROMA: A robust ICA-based strategy for removing motion artifacts from fMRI data. Neuroimage 112, 267-277 (2015).

54. R. H. R. Pruim, M. Mennes, J. K. Buitelaar, C. F. Beckmann, Evaluation of ICA-AROMA and alternative strategies for motion artifact removal in resting state fMRI. Neuroimage 112, 278287 (2015).

55. J. W. Szopinska, et al., Reliability of a participant-friendly fecal collection method for microbiome analyses: a step towards large sample size investigation. BMC Microbiology 18 , 110 (2018).

56. J. M. S. Fernández-Calleja, et al., Non-invasive continuous real-time in vivo analysis of microbial hydrogen production shows adaptation to fermentable carbohydrates in mice. Scientific Reports 8, 1-16 (2018).

57. J. Ramiro-Garcia, et al., NG-Tax, a highly accurate and validated pipeline for analysis of $16 \mathrm{~S}$ rRNA amplicons from complex biomes. F1000Res 5, 1791 (2018).

58. J. Szopinska-Tokov, et al., Investigating the Gut Microbiota Composition of Individuals with Attention-Deficit/Hyperactivity Disorder and Association with Symptoms. Microorganisms 8, 406 (2020).

59. P. J. McMurdie, S. Holmes, phyloseq: An R Package for Reproducible Interactive Analysis and Graphics of Microbiome Census Data. PLOS ONE 8, e61217 (2013).

60. R. Choudrey, "Variational Methods for Bayesian Independent Component Analysis," University of Oxford.

61. Llera A., Linked ICA in HCP500. GitHub (2019). 